\title{
Detección de cardiopatía en escolares chagásicos y no chagásicos de Combarbalá, IV Región, Chile
}

\author{
Ximena Aguilera M. '; Arruro Arribada C. ${ }^{2}$; Werner Apt B.'; \\ Inés Zulantay A. ${ }^{1}$; Jorge Rodriguez T.'

\section{Electrocardiographyc signs of cardiomyopathy in school children with serological evidence of Trypanosoma cruzi infection}

\begin{abstract}
Cimical, anc serologic studies ror Chogos disease and for electrocordiographyc evidence of myocordial ab-

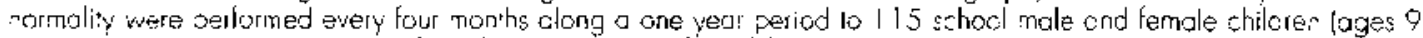

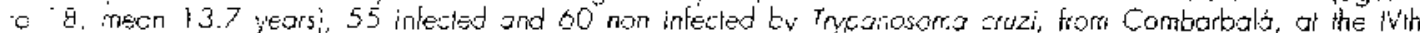
iCoaumboi Regio-. Cie. All subecls were symptom free and initia serologic stalus leither positive or negative indrect immunotuorescense. irdirect hemaglutination and ElISA recctions for trpancsomiasis| did not change in anycne of them along the whole study period. nevertherless thei elscirocardicgrpphyc records sniowec zvidence included for chagosic cardiotivopahy by PATO criterio in 22 [40\% of infected shildren Mcsl frequent electrocardiograshve asmormal ties in these polienis were prolonged $Q T$ inlerval and ncomplere right buncle bannth block. Electrocodfiographyc signs trat were not consicered anong PA.HO criteria to: suspectec chagasic cardiaryopathy were de ecled i? $7.3 \%$ and $6.7 \%$ of intected ond non intecied children respectively. Ninery one

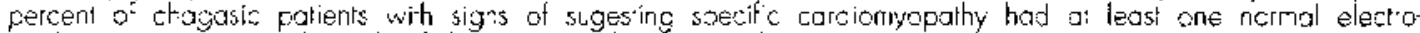
cardiogram sometime along the fellow-up peried. This los finding was not seen in petierits considerec to have ccrdiopalhy of another ethiclogy.
\end{abstract}

¿Key words: C-agas disease, Ifyponosomo brozı, soulh amer can trypcnosomiasis, cordionyopahy, electrocar diograr. i

En Chile. la forma de presentación clínica mis frecuente de la enfermedad de Chagas es la cardiopatía, le siguen en frecuencia el megacolon. a mayor distancia las formas congénitas y por último la acalasia'. Se ha estimado que $30 \%$ de los pacientes crónicos con enfermedad de Chagas en este país sufren de cardiopatía atribuible a dicha parasitosis ${ }^{2-8}$. Este porcentaje corresponde aproximadamente a 100000 personas de distintas regiones del área endémica de Chile. en especial habitantes rurales de la III y IV regiones. de Atacama y Coquimho respectivamente, consideradas hiperendémicas ${ }^{7-2}$. Aun cuando la mayoria de las personas con enfermedad de Chagas son asintomáticas, la cardiopatía chagrsica es crónica, progresiva. debilitante e

1. Departamento de Salud Pública Sur, Unidad de Parasitología. Faculıad de Medicina, Universidad de Chilc.

2. Departamento de Medicina Centro. Universidad de Chilc. Servicio de Medicina. Lnidad de Cuidados [orensivos. Hospital Clínico San Borja-Artiarán. incapacitante, y el riesgo de muerte de las personas afectadas, independientemente de tener o no síntomas, es más del doble que en las no infectadas ${ }^{8-10}$.

La investigación que se expone tuvo por finalidad conocer la frecuencia de cardiopatía en escolares de zonas hiperendémieas, un grupo poblacional antes no considerado. Con este propósito sc evaluaron clínica y electrocardiográficamente niños y adolescentes de Combarabalá, IV Región, en quienes se habian realizado previamente estudios serologicos para detectar enfermedad de Chagas".

\section{Material y Métodos}

Entre un tocal de 812 tscolares de enseñanza básica y media de Combarbalá estudiados serologicamente en una investigación reciente ${ }^{\prime \prime}$. se pesquisaron 55 niños -28 varones- con reacción de hemaglutinación indirecta (HAI)li inmunofluorescencia indirect (IFl, ${ }^{13}$ y ELISA IgG ${ }^{\text {IA }}$ positivas para la enfernedad de Chagas, cuya edad fluciuaba entre 9 y 18 años, promedio 13,8 años. Ellos constituyeron 
el grupo A, pacientes, de la presente investigación. El grupo B, controles, estaba conslituids por 60) niños, 31 vaco. nes, cuyos exńincmes serológicos dieron resuitados normales, de edades que lluctuabin entre 10 y 18 años, proniedio I4 años. Los controles futron seleccionados aleatoria ment: considerando su ctad. sexo y escolaridad para oble. ner una distribución similar que con los del grupo A.

En lodos los niños se realizó, al inicio del esiadio y cada 4 meses hasta completar 12 de seguimicinto, examen clinico. electrocardiograma (ECG) de 12 derivaciones (con registro lajgo de D2 para estudio de arritmias! y obteución de muestra de sangre venosa ( 5 a 8 mil para reaccioncs de HAI. IFI y ELISA para enfermedad de Chagas. En otras palabras, cuatro controles serolósicos, clinicos y electrocardiográficos a cada uno. Los trazados del ECG Juston interpretados a ciegas y de acuerdo a los criterios propuestos por la Organización Pananericana de la Salud (UPS para lo cardioparía chagásica crónica ${ }^{15}$, por uno de los autores (AAC), experto que desconocía los antecedentes it́nicos y el resulando de los exámenes serológicos de cuda niño. Los resulados fueron somelidos a pruebas de chi cuadrado. de tendencias $;$ de proporciones $1 "$.

\section{Resultados}

Los 115 escolares eran asintomáticos. con presión arterial y examen físico normal y se mantuvieron aparentemente sanos hasta el final del estudio. Ninguna de las Ires reacciones serológicas efectuadas a cada niño mostró variación en alguno de ellos en el transcurso del estudio. de modo que los 55 previamente positivos (grupo A) y los 60 negalivos (grupo B) siguicron siéndolo en los diferentes controles.

Los resultados de los electrocardiogramas clasificados como nomal o alterado se muesIran en la tabla 1. En 22 (40\%) de los escolares (13 varones) con reacciones serológicas positivas para enfermedad de Chagas se registraron alteraciones electrocardiograficas incluidas on los criterios sugerentes de cardiopatía atribuible a la parasitosis ( $\mathrm{p}<0,00001$ ); el promedio de sus edades fue 13,7 años.

La frecucncia de 1razados electrocardiográficos con alteraciones no atribuibles a cardiopatía chagásica, de acuerdo con los criterios aplicados, fue similar $(p>0,05)$ en los niños del grupo $\mathrm{A}$, donde se registraron en cuatro varones (7.3\%) de 13,2 años de edad en promedio y en los niños controles, dos varones y dos mujeres (6.7\%) cuya edad. promedio, era 12,8 años (tabla (). El detalle de los hallazgos electrocardiográficos en los niños de los grupos A y B se expone en la tabla 2. Las alteraciones más frecuentes en el grupo A fueron: QT prolongado (bloqueo incompleto de rama derecha del haz de
Tabla 1

Resultado del electrocardiograma en nirĩos de Combarbalá, Chile, con scrología positiva y negativa para enfermedad de Chagas en 115 escolares

\begin{tabular}{|c|c|c|c|c|c|c|c|}
\hline \multirow{3}{*}{$\begin{array}{l}\text { Serología } \\
\text { Chagas }\end{array}$} & \multicolumn{7}{|c|}{ Electrocardiograma } \\
\hline & \multicolumn{2}{|c|}{ Normal } & \multicolumn{2}{|c|}{ Alterado } & \multicolumn{3}{|c|}{ Alterado" } \\
\hline & $\mathbf{n}$ & $\%$ & $\mathbf{n}$ & $\%$ & $\mathbf{v}$ & $\%$ & $\mathbf{n}$ \\
\hline Positiva & 29 & 52.7 & 22 & 40,0 & 4 & 7,7 & 55 \\
\hline Negibiva & 56 & 93.3 & (1) & 0,0 & 4 & 6,7 & 60 \\
\hline
\end{tabular}

* Atheración alribujble a la enfermedad de Chagas

** Alceración no atribuible a la enfermedad de Chapas

\section{Tabla 2}

Hallazgos electrocardiográticos en escolares con reacciones séricas positivas y negativas para enfermedad de Chagas

ECG:
Condiction

Grups A Grujo B Chagas Controt (n: 55) (n: 60\}

\section{Arritmias auriculares}

Hipenrofia septal + disociación AV isor. $\quad 1^{\mathrm{k}} \quad 0$

Hipertrofia scptal + extrasistolia

$I^{*} \quad 0$

Trastornos de conducción AV

Hipertrofia Aul + bloquco AV gr I L I*

\section{Arritmias ventriculares}

Parasístol: + QT prolongado

Exlaasictolia + BIRD

10

Trastornos de conducción

BIRD

HBAI

HBAI + BIRD

HBP] + QT prolongado

$\mathrm{HBP}+$ + BIRD

HBP] + BCRD

$\begin{array}{ll}5 & 1 \\ 3 & 1 \\ 1 & 0 \\ 0 & 1 \\ 0 & 1 \\ 1 & 0\end{array}$

Otras alteraciones

Hipcrtofín septal + BIRD J* 0

QT prolongado

I) 0

Tofal alteraciones

264

"Alteración no atribuible a la enfermodad de Chogas

AV : auriculo-ventricular: grl: priner grado; isor: isorí́mica.

HB : heniblogueo; Al: anterior izquíerdo; PI: posterior izquicrdo;

BIRD: bloqueo incompleto de mana derecha del haz de Hiss:

BCRD: bloqueo completo de rama derecha: Aul: aurícula izquicrda. 
Hiss (BIRD) y hemibloqueo anterior izquierdo (HBAI). Se encontró diferencias significativas con respecto al grupo control en la frecuencia de prolongación de QT y BIRD ( $<<0.05$ ); no así en la de HBAJ ( $p>0,05)$. De los 22 escolares seropositivos en que se registraron alteraciones sugerentes de cardjopatía chagásica, éstas se detectaron en 12 casos en el primer electrocardiograma, en 9 desde el segundo y en uno desde el tercer trazado, siendo normales, en estos dos últimos casos, los registros precedentes. Sólo en dos de los 22 escolares con alteraciones cléctricas presumiblemente chagásicas no se registracon varjaciones en los diferentes controles electrocardiográficos (uno con hemibloqueo izquierdo anterior $y$ bloqueo incompleto de rama derecha y otro con hemibloqueo izquierdo posterior y bloqueo completo de rama derecha. En los veinte casos restantes, a lo menos uno de los cuatro trazados fue normal (tabja 3 ). Las alteraciones de los electrocardiogramas del grupo control, así como las de los cuatro escolares del grupo A no incluidas entre las atribuibles a la enfermedad de Chagas, no experimentaron modificaciones en todo el estudio.

\section{Comentario}

El análisis electrocardiográfico de los niños infectados revelo que una proporción significativa de ellos presenta alteraciones incluidas entre los criterios de sospecha de cardiopatía chagásica, lo que no necesariamente implica que la sufran, pues -por ejemplo- la imagen de bloqueo incompleto de rama derecha del haz de Hiss contemplada entre ellos, no es necesariamente anormal en los niños y en una proporción importante de mujeres jóvenes ${ }^{15}$. En todo caso el alto porcencaje de trazados alterados resulta alarmante si se considera que se trata de niños con un promedio de edad de 13,7 años, en que la severidad de las alteraciones electrocardiográficas, si efectivamente corresponden a miocardiopatía chagásica, pudiese aumentar en el transcurso de los años, y con ella el riesgo de muerte súbita o la necesidad de implantar marcapasos $7-8$.

La prolongación del intervalo QT, la alteración más frecuente en el electrocardiograma de los pacientes infectados con Trypanosoma cruzi de esta serie, aparece más a menudo en los tra-

Tabla 3

Cambios en el electrocardiograma, incluyendo registros normales, en 20 escolares con serología positiva para enfermedad de Chagas y signos electrocardiográficos de miocardiopatía a lo largo de 12 meses de seguimiento

\begin{tabular}{|c|c|c|c|c|c|c|}
\hline \multirow{2}{*}{$\begin{array}{c}\text { Caso } \\
\mathbf{N}^{\circ}\end{array}$} & \multirow[t]{2}{*}{ Sexo } & \multirow{2}{*}{$\begin{array}{l}\text { Edad } \\
\text { (añus) }\end{array}$} & \multicolumn{4}{|c|}{ Eleclrocardiograma } \\
\hline & & & 1 & 2 & 3 & 4 \\
\hline 1 & $\mathrm{~F}$ & 15 & QT & QT & $\Lambda$ & QT \\
\hline 2 & $\mathrm{~F}$ & 14 & BIRD) & BIRD & $\mathrm{N}$ & BIRD \\
\hline 3 & $\mathrm{~F}$ & 18 & $\mathrm{~N}$ & QT & $\mathrm{N}$ & $N$ \\
\hline 4 & $\mathrm{~F}$ & 16 & $N$ & $\mathbf{N}$ & QT & QT \\
\hline 5 & $M$ & 16 & $\mathrm{~N}$ & HBAI & HBAI & $\mathrm{N}$ \\
\hline 6 & $M$ & 18 & $\mathrm{~N}$ & $\mathbf{P V}+\mathbf{Q T}$ & QT & QT \\
\hline$?$ & $\mathrm{~F}$ & 13 & $Q T$ & $\mathrm{~N}$ & $N$ & $\mathrm{~N}$ \\
\hline$B$ & $\mathrm{~F}$ & 12 & QT & $N$ & $\mathrm{~N}$ & QT \\
\hline 9 & $\mathrm{~F}$ & 12 & BIRD & $\mathrm{N}$ & $\mathrm{N}$ & $\mathrm{N}$ \\
\hline 10 & $M$ & 14 & BIRD & BIRD & BIRD & $N$ \\
\hline 11 & $M$ & 13 & QT & $\mathrm{N}$ & HBAI & HBAI \\
\hline 12 & $\mathrm{~F}$ & 11 & $\mathrm{~N}$ & QT & $\mathrm{N}$ & QT \\
\hline 13 & $\mathrm{~F}$ & 14 & $\mathrm{~N}$ & QT & $\mathrm{N}$ & $N$ \\
\hline 14 & M & 14 & BIRD & BIRD & $\mathrm{N}$ & BIRD \\
\hline 15 & $M$ & 13 & HBAI & QT & $N$ & $\mathrm{~N}$ \\
\hline 16 & $M$ & 12 & $\mathrm{~N}$ & QT & $\mathrm{N}$ & $\mathrm{N}$ \\
\hline 17 & $\mathrm{~F}$ & 14 & QT & $\mathrm{N}$ & $\mathrm{N}$ & $\mathrm{N}$ \\
\hline 18 & $M$ & 15 & $\mathrm{~N}$ & QT & $\mathrm{N}$ & $\mathrm{N}$ \\
\hline 19 & $\mathrm{~F}$ & 10 & $\mathrm{~N}$ & BIRD & $\mathrm{N}$ & BIRD \\
\hline 20 & $M$ & 12 & $\mathrm{~N}$ & $E V+B I R D$ & BIRD & BIRD \\
\hline
\end{tabular}

N: normal; QT: QT prolongado; BIRD: bloqueo incompleto de rama derecha; HBAI; hemibloqueo anterior izquierdo: PV: parasístole ventricular: EV: cxtrasistolía ventricular. 
zados que cambian de lo normal a lo patológico, y desaparece en el transcurso del tiempo ${ }^{7}$, haciéndose menos frecucnte a medida que se estudian pacientes de más edad, lo que sugiere que pudiese corresponder a la primera manifestación de afectación del miocardio y ser atribuible a algún proceso inflamatorio perivascular coronario que después se extingue.

Es muy importante destacar que en el grupo de escolares con reacciones serológicas positivas a la parasitosis, también se cncontraron alteraciones electrocardiográficas no atribuibles a aquélla, las que se mantuvieron estables, rcafirmando la suposición de que obedezcan a causas ajenas a la tripanosomiasis (congénitas o de ouro tipo). En los pacientes chagásicos, por lo general las alteraciones electrocardiográficas son cambiantes porque las lesiones son evolutivas?. Más aún, el electrocardiograma puede pasar por períodos de normalidad o blanqueo, que no im. plican mejoría de las lesiones del miocardio. A la larga se produce daño definitivo que conduce a bloqueo aurículo-ventricular de tercer grado (completo) o arritmias graves, algunas de ellas dependientcs de postpotenciales que facilitan la Cibrilación ventricular y la muerte súbita.

La ausencia de síntomas en los escolares infectados por Trpamosoma cruzi y con signos de miocardiopatía en el elcctrocardiograma. resalta la importancia de éste para el diagnóstico de esta afección, lo que ha sido también señalado en trabajos previos, como indicador fidedigno de la enfermedad en ausencia de otras manifestaciones tangibles ${ }^{7.8}$. Sin embargo, puesto que en la mayoría de estos escolares se obtuvo a lo menos un trazado de características normales a lo largo de un año de seguimicnto, en personas infectadas por $T$. cinzi es recomendable realizar un mínimo de tres regisiros seriados para establecer o descartar el diagnóstico de cardiopatía, en especial si se trata de niños.

\section{Resumen}

Con el propósito de registrar la aparición de alteraciones electrocardiográficas consideradas sugerentes de miocardiopatía chagásica se realizó un scguimiento clínico. electrocardiográfico y serológico (con reacciones de inmunofluorescencia jndirecta, hemaglutinación indirecta y ELISA para enfermedad de Chagas), cada cua- tro meses hasta completar un año de seguimien10, en 15 escolares asintomáticos de Combarbalá (IV Región de Coquimbo), 55 con evidencia serologica de enfermedad de Chagas y 60 sin ella, de ambos sexos y de 9 a 18 años de edad (media 13.7 años). Los electrocardiogramas mostraton signos incluidos en los criterios de sospecha de miocardiopatía chagásica de la OPS en 22 (40\%) de los escolares infectados por Trypanosoma cruzi, aunque todos continuaron asintomáticos por todo el seguimiento. Las alteraciones más frecuentes en ellos fueron prolongación del intervalo QT y bloqueo incompleto de la rama derecha del haz de Hiss. En $7,3 \%$ y $6,7 \%$ de los escolares infectados y no infectados por Tripanosoma cruzi respectivamente, se detectaron alteraciones electrocardiográficas consideradas no atribuibles a la parasitosis se. gún los criterios empleados. En 91\% de los niños infectados y con alteraciones electrocardiográficas sugerentes de cardiopatía chagásica se registró, sin embargo, a lo nienos un electrocardiograma normal, en cambio éste estuvo siempre alterado en las niños cuyas anomalías tenían, supuestamente. otro origen.

(Palabras clave: enfermedad de Chagas, Tryamosoma cruzi, tripanosomiasis sudamericana, miocardiopatia, electrocardiograma.)

\section{Referencias}

1. Am W: Aspectos clínicos de la enfemedad de Chagas y sus repercusiones cconómicas. Programa de actividivdes. Taller sobre eradicación o control de la enfermedad de Chagas en Chile. Santiago, 1991:8.

2. Arribuda A. Apr W. Ugerte JM. Sundotal J: Cardiopatía chagásica en el valle de Elqui. Estudio epidemiológico y electroeardingráfico. Rev Med Chil 1979: 107 (9) -15

3. Ap W, Arribeder A, Arribade AM. Sandoned I. Ugerste IM: Cardiopatía chagásica en el vaile de Elqui. Estudio epidemiológíco, clínico y electrocardiográfico Kev Med Chil 1980; J08: 203-209.

4. Arribuded A, Ajn W. Amibada AM. Sundowed I: Cardiopatía chagńsica en la provincia de Chañaral. Rev Med Chil 1980: 108: 1118-1124

5. Ap W, Arribuda A, Ugarte JM, Sandoval J. Arribada AM: Cardiopatía chagásica en la IV región. Estudio clinico epidemiológico en las localidades de Salamanca, Combarbalá a lllapel. Rev Med Chil 1981: 109: 197-205.

6. Apr W. Arribada A. Sagta H. Gonzallez I. Araya J: Cardioparia chagásica en el altiplano chileno. Estudio elínico, epıdemiológico y electrocardiográfuco. Rev Hed Chil 1987: 115:616-623. 
7. Arribida A, Apt W, Ugarie M: Evolución de la cardinpatía thagásica durante un períndo de cuatro años en un grupo de pacientes chilenos. Bol of Sanit Pauan 1987; 102: 49- 72 .

8. Arribuda A, Ap' W. Aguilera $X$, et at: Cardioparía chagásica en Chile. Cardiol Intercon 1993; 2 : 94-99.

9. Apr W. Reyes $H$ : Aspectos epidemintógicos de la enfermedad de Chagas en Chile. l: distribución gcográfica. índices de infección en vectores $y$ en humanos. Parisitol al Día 1986; 10:94-101.

11). Schmunir $G A$ : La tripasosomiasis americana cono probletna de salud pública. Capítujo I del libro "La enfermodad de Chagas y el sistema ne[r]iso". O.P.S. Publicación cientílica 547. 1994:3-31

11. Agwitera S. Apt W. Redríguez J. et at: Eficacia del control de] vector de la enfermedad de Chagas demostrada a lravés de la infección humana. Rev Med Chil 1994: 122: 259-264.
12. Knierim fi, Sandovth J. Muñoz E: Reaccion de hemaglutunaciôn indirceca en enfernedad de Chagas crónica. Bol Chile Parasitol 1973: 28: 54-57.

13. Camorge ME: Fluorescence antibody test for the serodiagnosis of American typanosomiasis. Technical modification employing preserved culture forms of Trupermosmut crusi in slide tests. Rev Inst Med Trop Sau Paulo 1979; 8: 227-234.

14. Voller A: Microplate enzyme linked inmunosorbent assay for Chagas disedse. Lancer 1975: 1: 426-428.

15. Nimenclatura: Críticu diagnóstico electrocardio gráfico en la cardiopatía chagásica crónita. Prograna de salud humana B.I.D. Ministerio de Salud, Universidad del Salvador 1985; 5-28.

16. Stuchisn J, Clarek CH. Cau-Garcít $R$ : Principios y melodos estadisticos para comercio y economía. South-uesiem Publisbing Co: Cincinnati, Ohio, USA 1980.

\section{AVISO A LOS AUTORES}

Con el objeto de acelerar la edición de los trabajos se solicita encarecjdamente a los autores que disponen de computador con procesadores de texto IBM compatibles, enviar -en lo posible junto con las dos copias impresas de su material-, otra en disco flexible (tamaño 3,5" o 5,25", de doble o alta densidad) del mismo texto. El disco será devuelto de inmediato por correo certificado o por mano al autor corresponsal. 